\title{
Espectroscopia de prótons por ressonância magnética
}

\author{
Claudia da Costa Leite
}

A espectroscopia de prótons vem sendo utilizada nas áreas acadêmica e industrial desde a década de 60, no entanto, sua aplicação na avaliação bioquímica do cérebro in vivo é mais recente.

A espectroscopia por ressonância magnética e a ressonância magnética "convencional" são métodos que se utilizam dos mesmos princípios físicos, diferindo na forma em que os dados são processados e apresentados. Em vez das imagens anatômicas de ressonância magnética a que estamos acostumados, as imagens de espectroscopia são representadas por um gráfico, que demonstra picos de metabólitos que apresentam diferentes radiofreqüências e intensidades.

A espectroscopia pode ser obtida a partir de diversos átomos como hidrogênio, fósforo, carbono, sódio e flúor. De maneira geral, do ponto de vista clínico, a espectroscopia mais utilizada é a de hidrogênio, devido à abundância deste átomo no organismo. A espectroscopia de prótons permite a distinção entre tecidos normais e anormais.

A espectroscopia de prótons analisa amostras tridimensionais ("voxel"). De acordo com o "voxel", a espectroscopia pode ser dividida em "voxel" único e "voxel" múltiplo.

Existem duas técnicas básicas de realização de espectroscopia de prótons: "stimulated echo acquisition mode" (STEAM) e "point resolved spectroscopy" (PRESS), que variam de acordo com os parâmetros escolhidos para a obtenção do espectro.

Para que uma substância seja detectada pela espectroscopia de prótons, sua concentração deve ser maior que $0,5-1,0 \mathrm{mmol} / \mathrm{l}$. Por esse motivo, a maioria dos neurotransmissores e aminoácidos essenciais não é detectada por esta técnica, sendo então detectadas outras substâncias chamadas de metabólitos, cujo papel no cérebro muitas vezes ainda é desconhecido ${ }^{(\mathbf{1})}$.

Os metabólitos identificados pela espectroscopia são: $\mathrm{N}$-acetil-aspartato, colina, creatina total, mio-inositol, glutamato/glutamina, lactato e lipídios ${ }^{(2)}$. Em geral, os metabólitos não podem ser estudados em valores absolutos e sua avaliação é feita por meio de relações, sendo, em geral, o denominador a creatina ou, menos comumente, a colina. Ainda deve-se saber que a concentração dos metabólitos varia de acordo com a localização no encéfalo e com a idade do paciente ${ }^{(3)}$.

Algumas indicações já estão bem estabelecidas: diferenciação entre lesão focal neoplásica e não-neoplásica, diferenciação entre radionecrose e recidiva tumoral, diagnóstico de doença de Canavan e detecção de encefalopatia hepática subclínica ${ }^{(4-6)}$. Outras aplicações estão sendo pesquisadas exaustivamente. 
A grande discussão está em quais as verdadeiras indicações e aplicações da espectroscopia de prótons, pois como qualquer método novo, num primeiro momento, parece ao menos tentar resolver alguns dos dilemas diagnósticos, antes não-resolvidos pelas outras técnicas que já utilizávamos.

\section{REFERÊNCIAS}

1. Kwock L. Localized MR spectroscopy. Basic principles. Neuroimaging Clin N Am 1998;8:713-31.

2. Castillo M, Kwock L, Scatliff J, Mukherji SK. Proton MR spectroscopy in neoplastic and non-neoplastic brain disorders. Magn Reson Imaging Clin N Am 1998;6:1-20.

3. Pouwels PJW, Frahm J. Regional metabolite concentrations in human brain as determined by quantitative localized proton MRS. Magn Reson Med 1998;39:53-60.

4. Nelson SJ, Huhn S, Vigneron DB, et al. Volume MRI and MRSI techniques for the quantitation of treatment response in brain tumors: presentation of a detailed case study. J Magn Reson Imaging 1997;7:1146-52.

5. Austin SJ, Connelly A, Gadian DG, Benton JS, Brett EM. Localized 1H NMR spectroscopy in Canavan's disease: a report of two cases. Magn Reson Med 1991;19:439-45.

6. Danielsen ER, Ross BD. Magnetic resonance spectroscopy. Diagnosis of neurological diseases. Califórnia: Marcel Dekker, 1999. 